\title{
Representations of the Future in Depression-A Qualitative Study
}

\author{
Ali Sarkohi ${ }^{1,2}$, Karin Forslund Frykedal ${ }^{1}$, Hazel Holmberg Forsyth ${ }^{2}$, \\ Staffan Larsson ${ }^{1}$, Gerhard Andersson ${ }^{1,3}$ \\ ${ }^{1}$ Department of Behavioural Sciences and Learning, Linköping University, Linköping, Sweden \\ ${ }^{2}$ Department of Psychiatry, Linköping University Hospital, Linköping, Sweden \\ ${ }^{3}$ Section of Psychiatry, Department of Clinical Neuroscience, Karolinska Institute, Stockholm, Sweden \\ Email: ali.sarkohi@liu.se
}

Received January $14^{\text {th }}$, 2013; revised February $16^{\text {th }}$, 2013; accepted March $12^{\text {th }}, 2013$

\begin{abstract}
Copyright @ 2013 Ali Sarkohi et al. This is an open access article distributed under the Creative Commons Attribution License, which permits unrestricted use, distribution, and reproduction in any medium, provided the original work is properly cited.
\end{abstract}

\begin{abstract}
Previous studies indicate that the ability to imagine negative or positive future events affects psychological well-being and is a characteristic feature of depression. The aim of this study was to investigate how depressed individuals view their future during different time periods. A total of 15 individuals with a diagnosis of major depression were recruited from a psychiatry clinic and completed a semi-structured qualitative interview. Questions were asked about the situation right now, before becoming depressed, and the future (nearest time, within a year and the upcoming 5 - 10 years). Data were collected and analysed using a qualitative approach inspired by grounded theory. The results showed that depressed individuals experienced a state of "ambivalence", with negative cognitive, emotional, physical and socioeconomic consequences when they were asked to think about the nearest future. Ambivalence and its negative emotional and cognitive effects were substantially reduced in strength when they were asked about their more distant future. We conclude that the concept of ambivalence in depression and/or anxiety in the present may be an important feature of depression which deserves more attention from both a theoretical and clinical perspective. The use of qualitative approaches in the study of depression is encouraged.
\end{abstract}

Keywords: Future Thinking; Depression; Ambivalence; Time Horizons

\section{Introduction}

It is known that the ability to imagine negative or positive future events affect psychological well-being and can play an important role in the process of recovery, persistence and relapse of depression (MacLeod, Tata, Kentish, \& Jacobsen, 1997). A characteristic feature of many depressed individuals is a pessimistic and negative view of their personal future. This clinical observation is covered in several conceptualisations of depression (e.g., Beck, Rush, Shaw, \& Emery, 1979; Abramson, Alloy, \& Metalsky, 1989; Klinger, 1993). For example, Beck et al. (1979) described a cognitive triad, where depressed individuals have a negative view of themselves, the world and the future. Beck et al. argued that hopelessness about the future play an important role in depression. Hopelessness, in turn, was conceptualised by Abramson, Alloy and Metalsky (1989) as a deficit of positive expectancy and excess of negative projected thoughts. Macleod, Rose and Williams (1993) argued that the despair about the future is characterized by the lack of positive expectations, not necessarily by increased negative expectations. According to Klinger (1993), hopelessness in depressed persons often concerns future periods when the wished for goal seems to be out of reach (e.g., "I will never get married"). Melges and Bowlby (1969) argued that hopelessness is about reduced expectancy of success. In sum, several authors have argued the expectancies about the future is a central component in depression.

In the early 1990s, MacLeod and his colleagues investigated the significance of negative and positive expectations of the future. They pointed out that research to date has focused on the importance of negative thoughts about the future and devoted less attention to the importance of positive expectations. They further emphasized that positive and negative thinking has long been regarded as opposite poles on a single scale. Subsequent research has shown that positive and negative thinking rather should be regarded as two separate systems, where increase in one domain will not necessarily mean the decline in the other (MacLeod \& Moore, 2000). In order to measure future-oriented thinking MacLeod et al. (1993) developed the Future Thinking Task (FTT). The FTT is based on a verbal fluency task (Lezak, 1995), and the person is asked to generate as many positive and negative anticipated events as possible within one minute along various time periods, from the near future (within a week) to a longer period of time (next year and the next 5 - 10 years). Research on the FTT shows that parasuicidal individuals report fewer anticipated positive experiences than controls, but no overall increase anticipation of negative future experiences. The results for depressed and non-depressed parasuicides are essentially the same (MacLeod, Pankhania, Lee, \& Mitchell, 1997; Conaghan \& Davidson, 2002). Previous studies with depressed individuals show both an increase in expected negative events and a reduction of positive events on the FTT (McLeod \& Byrne, 1996). In a recent study, we found that depressed persons reported fewer positive future-oriented thoughts than the control group (Bjärehed, Sarkohi, \& Anderson, 2010). These findings indicate that a reduced anticipation of future positive 
events is a characteristic feature of depressed individuals even in the absence of suicidal ideation.

One limitation with the FTT is that the test may not capture the qualitative aspects of how depressed individuals perceive their future. First, the limited time given restricts the possibility to reflect and situate the expected future within a broader framework. Second, while the valence of the generated future expected events is scored (e.g., likelihood) it may still not be what the depressed person view as most important. Finally, as the FTT rely on cognitive function (i.e., speed of processing), some depressed may fail to generate future events on the FTT while still having thoughts about the future. As far as we know there is no qualitative study of depressed people's thoughts about the future in positive and negative sense, and along different time horizons, therefore, we adopted a qualitative approach. The aim of this study was to investigate the substantive content of representations of the future in depressed individuals by using open-ended methodology inspired by grounded theory. A further aim was to gain knowledge that could inform future investigations on the role of expectations about the future in depression.

\section{Methodology}

\section{Participants}

A sample was selected who were under treatment at a psychiatry clinic and volunteered to participate. A total of 15 informants were included. They all had been diagnosed with major depression using the DSM-IV (American Psychiatric Association, 2000). There were 8 women and 7 men, and ages ranged between 26 to 63 years. Participants differed in many respects. For example many were on sick-leave and some were unemployed. A few were inpatients due to earlier suicide attempts. Different educational levels and occupational backgrounds were represented, as well as differences regarding marital status. For most onset of depression occurred after puberty, but for a few the first episode debuted earlier.

\section{Interview Guide}

We prepared an interview guide inspired by Macleod et al.'s (1993) FTT, but only used the questions from the FTT and presented them in an open-ended version with no time constraints. The interviewer asked the participants what they thought was going to happen in the future and added some other questions, including a question about the time before the participants became depressed. More specifically, the interview guide included questions about how participants look at their situation right now, before they became depressed and how they saw their future (nearest time, about a year and the coming 5 - 10 years). Moreover, in an open-ended manner we added questions about how informants viewed their relatives' future and their thoughts about the future in Sweden, Europe and worldwide. Finally, they answered questions about whether they saw any obstacles and/or opportunities in their future. Any open question was followed by one or more, follow-up questions to get more detailed descriptions from the informants.

\section{Procedure}

Written and oral information about the study was provided to informants by the psychiatry staff. In addition to information about the purpose of the study etc., they provided data on researchers' names, phone numbers and email addresses in case someone would like to ask questions about the study. Both the written and oral data clearly revealed that participation was voluntary and confidential, and that the contents of the interview would not affect their on-going treatment at the clinic in any way. Those who gave informed consent all met the criteria of major depression using DSM-IV (American Psychiatric Association, 2000). Those with psychotic or manic symptoms, ongoing drug and/or alcohol abuse, current suicide plans and/or had been depressed before but not at the studied time were not asked to participate.

Included participants were contacted by a nurse to make an appointment for an interview at psychiatric clinic, Linkoping's university hospital. The participants completed a semi-structured qualitative interview which lasted between 45 to 60 minutes. Interviews were recorded and transcribed after the interview before the analysis. The interviews were conducted in a quiet room in the clinic. The interviewers were trained in quailtative interview, aimed to be empathic, showing a genuine interest in the participant, actively listened, and encouraged the participant to explore their thought by using relevance followup questions like: "would you tell me more about that?" The study was part of an on-going clinic based study on the treatment of depression for which ethical approval had been obtained.

\section{Data Analysis}

The analysis started after the first interview and continued simultaneously. Grounded theory (GT) methods (Glaser \& Strauss, 1967; Glaser, 1978; Charmaz, 2006) were conducted to collect and analyse data. The data collection process was guided by theoretical sampling, where data collection and analysis ran in parallel. By careful analysis, through substantive coding (open and selective), we developed an interpretive understanding of what the informants are occupied with and which was their main concern. The main steps during the analysis of data were coding (initial/open coding, focused/selective coding and theoretical coding), constant comparison, memo writing and memo sorting.

The data were constantly compared with codes (e.g., don't dare, fear, worry, threat, focus on now, shame, guilt, bubble, villain, anxiety, irresolute, apathetic, uncertain, pending, nowpast, now future, past, future, must, as-if, ruminate, suicide ideation/suicide attempt etc.), subcategories (e.g., thought, feeling, behaviour, oscillation, life line), categories (mood and time horizon), and core category (ambivalence). During the analysis we wrote down our ideas about relationships between the codes and other theoretical ideas that came to our mind, compared and sorted them (memo writing and sorting).

Theoretical coding (e.g. consequences, causes, effects, strategies, processes, etc.) was applied to understand how the substantive categories were related to each other, to the core category and to derive a theoretical model. Theoretical codes are used as tools to theorize the substantive codes (Stern, 2007). The important issues in this process have been to examine if the categories fit with the core category, subcategories, and how a theoretical model can be built based on comparative analysis between the data.

The open coding ended when the core category appeared. 
The theory was delimited to the core category. The core category became a guide for further questioning (Glaser, 1978). Follow-up questions in the last five interviews were theoretically sampled after the core category had emerged. The initial codes were integrated to conceptualize and theorize how the substantive codes and categories may relate to each other as a hypothesis to be transformed into a theory (Glaser, 1978).

In the analysis we aimed to adhere to the Strauss and Corbin (1998) principle of interaction between sensitivity (i.e. sensitive proximity - the ability to feel/read the subtle nuances and hints in the data) and objectivity (analytic distance-the distance between the empirical material and the emerging results). While two of the authors have a cognitive-behavioural orientation we tried, as much as possible, to keep an open mind (theoretical agnostic) (Charmaz, 2006; Dey, 1999; Glaser, 1978).

The authors and an external person (colleague who had not been involved in this study) compared the results and through an interactive process of discussion, analysis and review of the interview format, interview notes and the initial coding, arrived at consensus on a refined coding. Using this coding, the researchers independently coded the remaining interview notes and met to compare different perspectives and interpretations of ambiguous data and identification of concepts that were not covered by coding. Differences could be reconciled without any major disagreements. The researchers reviewed their notes and coding to ensure that no significant concepts had been overlooked, wrongly sorted or left uncoded. This resulted in coding of any additional statements with existing encodings but no new categories or disagreements with the encoding task.

\section{Results}

Participants' representations of the future along different time horizons (short and long term) are presented below, where ambivalence has been distinguished as core category. It is important to note that the participants' representation varied and expressed in different ways. The results are based on the common pattern, but sometimes there are quotes that clarify variation.

\section{Ambivalence}

Recurrently and in various ways informants described a state of ambivalence which was expressed in the following three domains: thoughts, feelings and behaviour. Shifting between choosing and not choosing, and to dare or not dare, took place in the three time dimensions: the past and the present, the present and the future with varying degrees of emotional, cognitive and behavioural responses.

Partly, there was an oscillation between past and present, where attempts to free themselves from the burden of the present had failed (even suicide attempts) and created disappointment and depression. The disappointment seemed to permeate the thoughts about the nearest future which lead to a generalization of perceived disappointments over time. When informants did not make any significant difference between the past and the possible future disappointments they became apathetic. In such situations, they neither wanted nor dare to think and believe in the future.

"Sometimes you feel like you have the future behind you. I'm not as aware of this development as I would like to be, because I have this "clinical picture”. ‥ I think that I sit sometimes and am quite lethargic, can't get on with it. And now the amount of Cipralex has increased from 15 mg to 40 , which the body has responded with a couple of side effects” (Male 57 years).

"I dare not think about the future because it never ends up like I figured it” (Int.: Okay).

“I suppose that is something I've learned on the way. You should stop thinking about the future because it is never ends as expected" (Female 44 years).

For some there was an oscillation between the present and the near future which gave an experience of "threat", "fear" and "worry". The fear of facing new disappointments was considered as the "villain of the piece", giving a sense of "going into a bubble" and becoming increasingly depressed. This gave a feeling of being "mentally handicapped", which created suicidal ideation and the thought that suicide was the only option. In addition to their depression and poor financial situation they were afraid of losing their social network and possible employment.

"The worst is this panic anxiety. The depression is a bit calmer. But that's it, you go into a bubble. If you'd ask me, you go very low, depressed or what should I say? $\cdots$ It's the thing with this panic anxiety and that. That is what the culprit is. You can have a depression even though you work. You sort of go in to your own little world, but you still work. I wonder what percentage of the Swedish population who suffer from depressions, but I haven't checked. It is probably fairly high, I think, but it's probably something "they can live with". (Int.: But in your case, there is the threat of getting sick again, as bad as you were?). Yes, I'm terrified $\cdots$ and I daily think "what will happen in 2 years?” I sit and worry about what will happen in 2 years $\cdots$ the authorities and people say "do this, do that" and decide what I should do when I become a different person. That is when I fear myself $\cdots$ I was very close to hang myself. Nice to just escape all this stuff” (Male 42 years).

In order to avoid anxiety and experience of "being in a bubble" the informants reported using various survival strategies (e.g. "here and now focus", "is and should world", "must-rules", "as-if-rate”, "suicide attempt” etc.). The strategies used varied depending on the time dimension.

\section{Ambivalence in Relation to Different Horizons of Time}

\section{Future Thoughts about the Near Future}

There was a desire to believe in oneself, one's future, to form an opinion, to make decisions and plan one's near future, but the informants reported that they "don't dare”, since "fear of not coping with my plans" raises "worry/anxiety of being disappointed". The near future was thus perceived as "threatening". Informants became immersed in negative thoughts and feelings about what and how things would be done. Implicit and/or explicit wishes and decisions raised fear/worry. In addition to fear and anxiety they felt "shame and guilt" for not having found and/or provided long-term and constructive solutions to their problems.

Ambivalence was evident and problematic when informants confronted a problem and had to come up with a solution. 
Overall, making decisions regarding the near future was sometimes difficult. In such a situation, they experienced simultaneous contradictory positive and negative thoughts and emotions, which made them behave increasingly uncertain, pending, irresolute and sometimes apathetic.

The informants would like to have the positive things that are associated with their choice and at the same time avoid the predictable negative consequences. It seemed as if the positive aspect of individual choice was overshadowed by the fear and worry of the expected negative consequences. This may make the individual switch between two positions, namely to dare to choose or not choose their future plans. In the short term they often described the fears and hopes in general terms.

“Many times I don’t dare. You become a bit $\cdots$ I am also like that in my personality that I $\cdots$ I want that familiarity, I want that sweet, nuclear family. eh.. oh.. I would like to have thoughts of the future where things will be great and to feel safe and to know whom you belong to, and so on. But, many times it will not be as expected, as I imagined and then I get so disappointed. (Int.: Okay). And it has ‥ I divorced three and a half years ago and one of the main causes for the divorce was that I was constantly disappointed, from a mere trifle to bigger issues. (Int.: But if we talk about the very near future. What are your thoughts on that?). I don't dare to think about the future since it will never be as I imagined any way. (Int.: Okay). That's something I learned along the way. You have to stop having thoughts of the future, it will never be as you expected anyway" (Female 44 years).

Ambivalence and well-being affected each other. Ambivalence could impair well-being which in turn exacerbated ambivalence. This created a vicious circle, making the depressed individual more and more uncertain, pending, irresolute and sometimes apathetic about making decisions about the near future. The consequence may be that informants "go into a bubble” or get stuck in a pre-existing bubble/depression.

"I can't directly plan from day to day, for it depends on exactly how I feel. $\cdots$ With depression and anxiety, you kind of get it for free $\cdots$ But with depression it has a lot to do with thinking. You think and live in a small bubble, and go in to one's own world and think; now I'll solve this-maybe you can't, but you're still thinking it again and again. So you go in to you little world and become depressed. Yes, you don't solve it and go deeper and deeper down. (Int.: You do not plan to $\cdots$ ). No, I don't dare. But if something might have happened. I'm not saying, I might kill myself tomorrow, I don't know. (Int.: You feel that unstable?). Yes. I can't directly plan from day to day, for it depends on exactly how I feel. But the worst is this panic anxiety. Depression is of course a bit calmer. But that's it, you go into a bubble. It is all about getting very low, depressed or how to say it. (Int.: But do you think it is how you feel that affects you that you are not able to think in the near future?) Yes, sure” (Male 42 years).

Depression and its adverse consequences affected the individual's ability to function both in social life and at work. It was considered as an obstacle for thinking about the near future. Depression lead to feelings of "guilt, shame" and "fear of being labelled" for life, and could lead the individual to "hide" the problem in social contacts.

"I feel, mostly when I am sick, there is a lot of shame. And even though there are many people who are depressed around, there's still a sense of shame anyway. To some, I can't say that I have a depression because it has a negative tone. Oh, and I have heard many of those who don't know that I have depression, say "yes, they will of course be labelled all their life" and it's like that $\cdots$ of course it worries me. $\cdots$ Who would want to employ someone who may be sick?” (Female 44 years).

Requirements and expectations from relatives, employers, insurance service, employment services, social services etc. were reported as affecting the informants negatively.

“The public employment service, social insurance, social services and society require a lot from me. Then I get panic anxiety $\cdots$ people say do this, do that, and decide what I should do, then I became another person. Then I get frightened of myself” (Male 42 years).

The situation created a dependency in relation to publicly funded health care and health insurance, which participants viewed as limiting the possibility of using their own resources to cope with everyday life and to have the courage to plan ahead. Sick leave and medication were described as necessary, but informants also said they would have preferred not to have the side effects of medication. They also disliked the prospect of a continued poor economic situation, unemployment, fear of being stigmatized, and be dismissed from the labour market due to mental illness.

Getting better was experienced as time-consuming and out of reach in the near future. Therefore different life strategies were used to avoid the uncertainty, fear and anxiety that were associated with decision making. In this mode, they reported they would use a "here and now" focus, "must rules" and "displace radical plans" further on in the future.

"I delimit it to cope with it, and it means I take one day at a time. I don't think, I get very stressed when I am thinking about the future ahead, because I'm still feeling bad. I feel bad in myself. I'm pretty handicapped, I call it mental paralysis. So I sort of think $\cdots$ today I'm coming here at two o'clock and then I'm going shopping. That's what I do today and then I have to look at what to do tomorrow, if it is anything I must do. Then I'll sit down tonight and think about it. (Int.: Then you start thinking about the next day?). Yes, basically if there is not anything you have to, like I know that I booked an appointment with my physiotherapist on Wednesday and it's only because I must cooperate, otherwise I'd rather decide at the same day. (Int.: But you take one day at a time for that?). To be able to handle it. Otherwise I start thinking about things that are threatening, that I should start working again, and then I panic because I am not ready for it” (Male 57 years).

The informants were aware about the importance of engaging in positive activities. The problem was that anxiety and ambivalences made it difficult to dare doing anything at all and lead to a feeling of living in two worlds namely "is and should world". Anxiety seemed to be "the villain of the piece" and something informants did everything to avoid. 


\section{Future Thoughts within a Year}

Even if one year is still perceived as a "too short time perspective” some informants dared to think forward, however in both positive and negative terms and in general and/or specific terms. Once again, they expressed fears and worry to be disappointed, but at the same time they expressed a cautious hope for a turnaround, where they could do some things (start work/study) and have good relationships.

"I have been ill for a long time and I have felt so terribly bad. You struggle just to survive the day and if you ask me "why do I do that?" if it still only gets worse. I think I ought to have crossed the border of pain, surely it can't get any worse” (Male 43 years).

"It was hard but I hope it is better and that I have come out of my depression and can live a normal good life. But I don't know, I hope so anyway. (Int.: What is a normal life for you?). Go out without fear and not feel stupid and being able to do stuff I can't do today, go to the store and things like that” (Female 26 years).

Despite some cautious hope informants expressed a fear of "not embracing change" and/or to "make mistakes". Furthermore, self-blame and self-devaluation occurred regardless of the fact that they realized that the environment had signalled other things than their own perceptions.

"I am, as they say sensitive to change so I think it's really hard to change; actually $\cdots$ there is an element of uncertainty." (Female 35 years).

"I want to move to Norway. ‥ Of course now, I need security and moving would be disastrous for me. Here, I have some friends in town and moving to a new environment would probably be dangerous for me. I would not be able to handle it now. (Int.: But if you feel better do you think this will come back to you, this future thinking and moving?). It was like having some dreams that I did not implement. Having some thoughts that one might $\cdots$ But those dreams, I never really lived them, it was more about what you could do with your future $\cdots$ it might be too big a change for me. (Int.: If you could live out those dreams?). Yes, I think so. I would take on more than I could manage. It's not just that, for wherever I move, I'll have no friends, no social environment, nothing to anchor to in the first place and one just has to work with that” (Male 43 years).

\section{Future Thoughts within 5 - 10 Years}

Informants were aware that depression had strong negative effects on their views of the near future, but in the long time perspective they hoped that depression would lead to some positive opportunities. In spite of chronic depression and experiences of previous unsuccessful treatments, there was a hope of and a longing for a therapeutic effect in the long run. In the long term they began both to wish and dare to think and plan ahead. Hope was often expressed in general terms, but sometimes in more specific terms. They felt more energetic and mentally on the way out of "the bubble".

"Yes (laughs). Then there would be a positive scenario in which I continue to be employed in any voluntary work and who knows, I might stumble on something that means a financial support in this. Or rather a contribution to the pension you to the normal retirement pension” (Male 63 years).

"I might dream. I've done it before. (Int.: What are you dreaming about?). I would like to move to Norway, partly because you make more money and partly because I love the culture there" (Male 43 years).

"Where I hope to be very happy, feel good, be like an ordinary person. And I would hope to have children, living an average Swedish life” (Female 31 years).

In the long term, informants were more willing and recaptive to major changes in both family and working life. They could for instance imagine that they would separate from someone and/or move in with someone, have children, go from sick leave to their former occupations (e.g., job/studies) or apply for new jobs.

It was not uncommon to condition their future positive thoughts by expressing themselves in "as-if" terms, which can be considered as another lifeline/survival strategy in the long perspective of time in the same way as 'must rules' in the limited time frame. Views on depression related to planning their own future, to learn from their life history and to help others. Informants occasionally told us that "there is a deeper meaning with the condition" and you should not only see the illness as a barrier but also to wonder about what positive things depression can bring.

"If I get well, I can help other depressed people, I can be more empathic and have a greater understanding of their condition $\cdots$ I will not bring my feeling, but bring experience” (Male 46 years).

Finally, some informants made no distinction between thoughts and feelings in a traditional way. Informants seemed to believe that feelings are to be considered as a form of thoughts, causing individuals with depression to feel that they are not able to solve problems. In other words, the problem in depression to some of them was the emotionally coloured thoughts which were seen as maintaining the depression (instead of rational thoughts).

"I would like to put it like this, there are two parts. There are these emotional thoughts, or what to call them. And then there are these more rational thoughts. But the rational thoughts are pushed away by the emotional, since they are so much stronger no matter what. Purely rational, I think that it doesn't need to be like this, it will resolve itself, and be this or that. But emotionally, I think that I will not be able to manage to arrange it to be just this and that" (Male 46 years).

\section{Model of Past and Future Thoughts about the Future}

In Figure 1 we describe a derived model of how the depressed informant may oscillate between thoughts about the future and thoughts about the past. In the near past the person may predominately remember "bad" things, and in the near future the person may also foresee aversive experiences and anxiety about the future. However, when thinking about the more distant future and past the emotional valence may decrease. These possible future experiences and recollections of good events in the past are however not accessed as the person wanders between the near past and the very near future. In therapy 


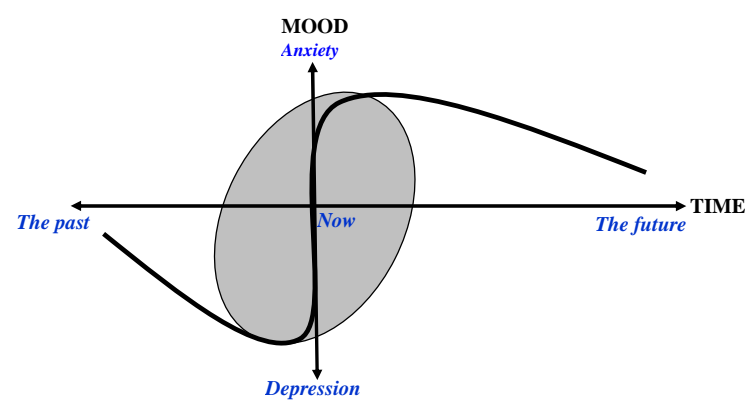

Figure 1.

Model of ambivalence. The strength of depression and anxiety varies as a consequence of the time perspective. Ambivalence is most present ("the bubble" i.e., gray zone), when depression and anxiety peak in the here and now.

it is common to focus on both past experiences and future planning and hence the person may be helped to overcome being stuck oscillating between the near future and the near past. The model shows that retrospective and prospective negative thoughts create and maintain anxiety and depression. Retrospective negative thoughts maintain depression and prospective negative thoughts create and maintain both anxiety and depression. As a way to avoid the discomfort associated with conflicting thoughts different survival strategies are applied depending on which time dimension they are in with their thoughts. Retrospective and prospective thoughts along different time dimensions may raise different types of emotional reactions. On the one side coexistence of both negative and positive prospective cognitions on the other side coexistence of both negative retrospective and negative prospective cognitions in the present moment without applying survival strategies seems to create ambivalence. This in turn may increase the risk for suicidal behaviour.

\section{Discussion}

The results of this study indicate that depressed individuals are experiencing a state of "ambivalence" with negative cognitive, emotional, physical and socio-economic consequences when they are asked to think about the nearest future. Ambivalence and its negative emotional and cognitive effects are substantially reduced in strength when they were asked to think in a longer time perspective such as 5 - 10 years.

Ambivalence creates a multifaceted picture where time is an important factor. This means that ambivalence is significant in the present moment in terms of thoughts, feelings and behaviours. Further into the future the ambivalence is reduced and worry also decrease. Thoughts about the distant future can even operate freely from ambivalence and anxiety. Ambivalence in the moment was created by a fear of being disappointed and once again fail. This ambivalence, caused by the fear of disappointment, is a seemingly insurmountable obstacle of getting out from the described "bubble". Ambivalence can lead to a feeling of hopelessness, which according to Beck et al. (1979) has an obstructive effect and plays an important role in depression and we suggest that it plays an important roll in anxiety as well. Hopelessness may be due to past memories, lack of positive future thoughts, or a surplus of negative future thoughts, but it might as well be due to a few qualitatively strong and persistent negative future thoughts. The ambivalent individual behaves uncertain, pending, irresolute and sometimes apathetic.
Several researchers have commented on ambivalence in depression. For example, the concept of "cognitive dissonance" (Festinger, 1957) can be applied, where one's thoughts/ideas are not consistent with each other, because the contradictory positive and negative thoughts appear almost simultaneously. This may consequently leads to a negative emotional and physiological resonance (emotionally and physically response in negative terms), with negative socio-economic consequences. Ambivalence has also been found in interpersonal relations, such as adult attachment (Busch, Rudden, \& Shapiro, 2004).

The intensity and frequency of the contradictory thoughts and individual internal/external resources to deal with these thoughts may be critical to the strength of emotions experience in depression. Oscillation between past and present, as found in this study, may be a useful strategy in the short run but instead of positive thoughts the depressed person may get stuck between anxiety and depression. In other words both thinking back and about the future may be a negative experience as the person fails to see beyond the very near present unless prompted. As a way to avoid past unpleasant memories and/or unpleasant threats in the future the depressed person may apply various survival strategies. The informants in this study reported that they were willing and able to think positively about their future, although in general terms, but that they were afraid to experience new disappointments. It is understandable that this situation often leads to avoidance. This may occur when an individual chooses to refrain from approaching a seemingly desirable situation in order to avoid experiencing any negative consequences that may be associated with it. It is a condition in which the individual perceives fear for something they wish. When the target is far away, both positive and negative feelings about the situation are less strong. Failing to regulate the cognitive dissonance can create disharmony and experience in the form of anxiety, guilt, shame, anger, insecurity, stress and other negative emotional states.

For the ambivalent depressed individuals the "here and now focus" becomes a lifeline and a choice of situations that do not require any immediate decisions. A disadvantage of this strategy, like other life-lines set out in the "must-rules" and "if-as" terms, is that the individual will not dare to think, plan and make decisions about future goals. Here and now focus will be of help only when the individual dare to set up concrete goals. However, in clinical situations it is always up to the individual to choose to expose themselves and strive towards their goals. It is interesting to note that the concept of mindfulness has been endorsed in the study of depression (Segal, Williams, \& Teasdale, 2002), but we believe that the strategies used in mindfulness (e.g. being in the present moment) are not the same as the "here and now" focus we saw in our informants which may be more motivated by avoidance. In literature it is often mentioned that depressed individuals have a pessimistic and negative view of the future, but the results of this study show that this perception may mainly only concern the very near future and that the distant future may not be viewed as negatively (e.g., Beck et al., 1979; Abramson, Alloy, \& Metalsky, 1989). This pessimism was apparently rooted in both the depressive thought content and the worry for the near future disappointments. Anxiety was said to be the "villain of the piece" and the ambivalence curve followed essentially the same curve as for depressive thoughts, i.e. the closer in time the more anxiety. While we separate anxious and depressive thoughts in this study, based on the reports from our informants, the current evidence strongly suggest that 
anxiety and depression overlap (Hirschfeld, 2001).

This study has limitations. First, the results were based on a limited number of interviews with volunteer participants who were recruited from an outpatient psychiatric clinic. While this may be enough to capture the phenomena of future beliefs, it is likely that other subgroups of patients, for example with less severe depression, fewer comorbid problems, and dropouts from treatment are not represented. A second limitation concern the risk that our group of informants, because of their current depressed state, were not able to provide full answers to the open questions. If this is correct informants may have more full representations of the future when feeling better. On the other hand, this is less of a problem for our study as informants had longstanding problems and not only a single episode of depression. Third, as with most research, our observations and conclusions are subject to potential bias from our preconceptions and prejudices. Finally, it is important to note that in this study, we have chosen to highlight the ambivalence from a cognitive perspective and in the interview guide explicitly asked informants about different time periods which might have not been discussed with a less structured interview. On the other hand, our findings are not markedly different from clinical reports on what depressed persons say about their future (Beck et al., 1979).

We conclude that qualitative approaches can be useful to add more knowledge on how depressed persons perceive their future and our hope is that the concept of ambivalence in depression and/or anxiety in the present gets more attention from both a theoretical and clinical perspective.

\section{Acknowledgements}

This study was sponsored in part from a grant by the Swedish Science foundation to the last author.

\section{REFERENCES}

Abrahamson, L. Y., Alloy, L. B., \& Metalsky, G. I. (1989). Hopelessness depression: A theory-based subtype of depression. Psychological Review, 96, 358-372. doi:10.1037/0033-295X.96.2.358

American Psychiatric Association (2000). Diagnostic and statistical manual of mental disorders (4th ed.). Washington DC: American Psychiatric Press.

Beck, A. T., Rush, A. J., Shaw, B. F., \& Emery, G. (1979). Cognitive therapy of depression. New York: Guilford Press.

Bjärehed, J., Sarkohi, A., \& Andersson, G. (2010). Less positive or more negative? Future directed thinking in mild to moderate depression. Cognitive Behaviour Therapy, 39, 37-45. doi:10.1080/16506070902966926

Busch, F., Rudden, M., \& Shapiro, D. (2004). Psychodynamic treatment of depression. Washington DC: American Psychiatric Publish- ing, Inc.

Charmaz, K. (2006). Constructing grounded theory. London: Safe Publications.

Conaghan, S., \& Davidson, K. M. (2002). Hopelessness and the anticipation of positive and negative future experiences in older parasuicidal adults. British Journal of Clinical Psychology, 41, 233-242. doi:10.1348/014466502760379208

Dey, I. (1999). Grounding grounded theory: Guidelines for qualitative inquiry. San Diego: Academic Press.

Festinger, L. (1957). A theory of cognitive dissonance. Evanston, IL: Row, Peterson.

Glaser, B. G., \& Strauss, A. (1967). The discovery of grounded theory: Strategies for qualitative research. New York: Aldine de Gruyter.

Glaser, B. G. (1978). Theoretical sensitivity. Mill Valley: The Sociology Press.

Hirschfeld, R. M. (2001). The comorbidity of major depression and anxiety disorders: Recognition and management in primary care. Primary Care Companion to the Journal of Clinical Psychiatry, 3, 244254. doi:10.4088/PCC.v03n0609

Klinger, E. (1993). Loss of interest. In C. G. Costello (Ed.), Symptoms of depression (pp. 43-62). New York: Wiley.

Lezak, M. (1995). Neuropsychological assessment (3rd ed.). New York: Oxford University Press.

MacLeod, A., Rose, G. S., \& Williams, J. M. G. (1993). Components of hopelessness about the future in parasuicide. Cognitive Therapy and Research, 17, 441-455. doi:10.1007/BF01173056

MacLeod, A. K., \& Byrne, A. (1996). Anxiety, depression and the anticipation of future positive and negative experiences. Journal of Abnormal Psychology, 105, 286-289. doi:10.1037/0021-843X.105.2.286

MacLeod, A., Pankhania, B., Lee, M., \& Mitchell, D. (1997). Parasuicide, depression and the anticipation of positive and negative future experiences. Psychological Medicine, 27, 973-977. doi:10.1017/S003329179600459X

MacLeod, A. K., Tata, P., Kentish, J., \& Jacobsen, H. (1997). Retrospective and prospective cognitions in anxiety and depression. Cognition and Emotion, 11, 467-479. doi:10.1080/026999397379881

MacLeod, C., \& Moore, R. (2000). Positive thinking revisited: Positive cognitions, well-being and mental health. Clinical Psychology and Psychotherapy, 7, 1-10. doi:10.1002/(SICI)1099-0879(200002)7:1<1::AID-CPP228>3.0.CO; $\underline{2-S}$

Melges, F. T., \& Bowlby, J. (1969). Type of hopelessness in psychopathological process. Archives of General Psychiatry, 20, 690-699. doi:10.1001/archpsyc.1969.01740180074007

Segal, Z. V., Williams, J. M. G., \& Teasdale, J. D. (2002). Mindfulness-based cognitive therapy for depression. New York: Routledge.

Stern, S. L. (2007). On solid ground: Essential properties for growing grounding theory. In A. Bryant, \& K. Charmaz (Eds.), The SAGE handbook of grounded theory (pp. 114-126). Los Angeles, CA: Sage Publication.

Strauss, A., \& Corbin, J. (1998). Basics of qualitative research (2nd ed.). Thousand Oak: Sage Publications. 